\title{
Effect of electron-phonon coupling on transmission through Luttinger liquid hybridized with resonant level
}

\author{
Alexey Galda, Igor V. Yurkevich and Igor V. Lerner \\ School of Physics and Astronomy, University of Birmingham, Birmingham B15 2TT, United Kingdom
}

(Dated: January 28, 2013)

\begin{abstract}
We show that electron-phonon coupling strongly affects transport properties of the Luttinger liquid hybridized with a resonant level. Namely, this coupling significantly modifies the effective energy-dependent width of the resonant level in two different geometries, corresponding to the resonant or antiresonant transmission in the Fermi gas. This leads to a rich phase diagram for a metal-insulator transition induced by the hybridization with the resonant level.
\end{abstract}

PACS numbers: $73.20 . \mathrm{Mf}$

Low-temperature electronic properties of onedimensional (1D) systems (like quantum wires or nanotubes) are strongly affected by electron-electron interactions. Electrons in such systems form a Luttinger Liquid (LL) 1]. An arbitrarily weak repulsion in a clean LL leads to power-law decay of various correlation functions with exponents depending on the interaction strength. Such a decay which is a characteristic property of the LL has been been experimentally observed in carbon nanotubes [2] and various quantum wires [3] (see Ref. 4 for a recent review).

Inserting a potential impurity or a weak link (e.g., a tunnel barrier) into the LL results in a power-law suppression of a local density of states (LDoS) at the impurity site and thus a suppression of the conductance at low temperatures $T$ [5, 6], changes in characteristics of the Fermi-edge singularity [7] and Friedel oscillations [8], etc. If the barrier interrupting the LL carries a discrete localized state resonant with the electron Fermi energy, its hybridization with the electronic states in the leads results in a sharp resonant transmission 9. Similar to the Fermi liquid, it is described by the Breit-Wigner formula but with the resonance width $\Gamma_{0}$ replaced by an energydependent effective width $\Gamma(\varepsilon)$ vanishing at the Fermi level. Such a resonant transmission can be realized, e.g., by inserting into a $1 \mathrm{D}$ quantum wire a double barrier with a resonant level or a weakly coupled quantum dot (QD) with sufficiently large level spacing $\delta\left(\delta \ll T, \Gamma_{0}\right)$ and one level in resonance. We would refer to this geometry as resonant-barrier.

In a dual geometry, when a QD with such a resonant level is side-attached to the LL, transmission becomes antiresonant: it is reflectance rather than transmittance which is described by the Breit-Wigner formula but with the width $\Gamma(\varepsilon)$ being power-law divergent at the Fermi level 10] (with the divergence cut by a temperature $T$ ). Both geometries are realistic: transmission and tunnelling measurements in the presence of controlled defects have already been performed in both quantum wires [3] and carbon nanotubes for various types of defects [11. Naturally in any realistic geometry electrons in the LL are inevitably interact not only with each other but also

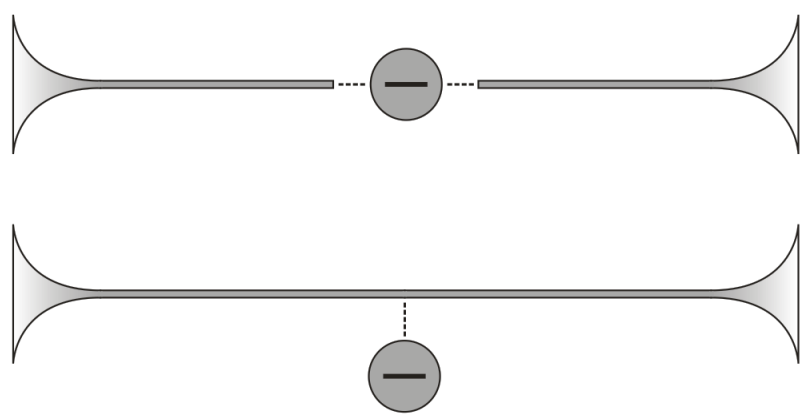

FIG. 1. The geometries considered: resonant-barrier geometry (top) and side-attached geometry (bottom), also referred to in the text as RBG and SAG.

with phonons.

It is known that the electron-phonon (el-ph) coupling in combination with the electron-electron (el-el) repulsion results in the formation of two polaron branches with different propagation velocities in the clean LL (see, for example, [12, 13]). The formation of polarons modifies the values of exponents in power laws characteristic for the LL. The exponents can change signs as functions of the relative strength of the el-el and el-ph coupling and of the ratio of the Fermi to sound velocities. The effect of this is especially pronounced for the LL with an embedded potential scatterer. While a single scatterer embedded into the phononless LL makes it going from an ideal metal to an ideal insulator (at $T=0$ ) 5 , in the presence of the el-ph coupling such a transition can be reversed for a weak scatterer [14] or, in general, can become dependent on the scatterer strength [15].

In this Letter we show that the el-ph coupling results also in a drastic change of electronic transport trough the LL hybridized with a resonant level both in the resonantbarrier and in the side-attached geometry (see fig. 1).

We will proceed as follows. First we introduce the action for the LL with the Coulomb repulsion, el-ph coupling and hybridization with a resonant level. By integrating out the phonon fields and the fields describing 
the resonant-level electron, we obtain an effective action in terms of only the fields describing the conduction electrons. Then we employ the functional bosonization in form developed in [16] to describe the polaron formation in the presence of the resonant level in terms of the mixed fermion-bosonic action. Finally we use the renormalization group (RG) analysis in form similar to that for the phononless LL [5, 10] to calculate transmission through such a polaronic liquid as well as the effect of the el-ph coupling and hybridization on the electronic LDoS in the vicinity of the QD.

\section{EFFECTIVE ACTION}

The action consists of three terms, $S=S_{\mathrm{LL}}+S_{\mathrm{el}-\mathrm{ph}}+$ $S_{\mathrm{T}}$. We assume the usual LL decoupling of the (spinless) electron field into the sum of right- and left-moving electrons,

$$
\psi(\xi)=\psi_{\mathrm{R}}(\xi) \mathrm{e}^{\mathrm{i} p_{\mathrm{F}} x}+\psi_{\mathrm{L}}(\xi) \mathrm{e}^{-\mathrm{i} p_{\mathrm{F}} x}, \quad \xi \equiv(x, t),
$$

with $\psi_{\mathrm{R}, \mathrm{L}}$ being labelled by $\eta= \pm 1$ below, and unit with $\hbar=1$ are used. Then the LL part of the action, which includes the electron kinetic energy and the density-density interaction, has the following form:

$$
S_{\mathrm{LL}}=\sum_{\eta= \pm 1} \int \mathrm{i} \xi \bar{\psi}_{\eta}(\xi) \mathrm{i} \partial_{\eta} \psi_{\eta}(\xi)-\frac{1}{2} \int \mathrm{i} \xi V_{0} n^{2}(\xi) .
$$

Here $n \equiv\left(\bar{\psi}_{\mathrm{R}} \psi_{\mathrm{R}}+\bar{\psi}_{\mathrm{L}} \psi_{\mathrm{L}}\right)$ is the electron density, $V_{0}$ is the screened Coulomb interaction, $\mathrm{i} \xi$ stands for $\mathrm{i} x \mathrm{it}$ and $\partial_{\eta} \equiv \partial_{t}+\eta v_{\mathrm{F}} \partial_{x}$. The free conduction electron Green function, $g_{\eta}\left(\xi-\xi^{\prime}\right)$, is defined by $\partial_{\eta} g_{\eta}\left(\xi-\xi^{\prime}\right)=$ $\delta\left(\xi-\xi^{\prime}\right)$ so that its Fourier transform is given by

$$
g_{\eta}(\varepsilon, q)=\left[\varepsilon+\mathrm{i} \delta \operatorname{sgn} \varepsilon-\eta v_{\mathrm{F}} q\right]^{-1} .
$$

We use here the zero-temperature formalism: the only role played by temperature is providing an alternative low-energy cutoff in the RG equations below.

It is not particularly important for what follows how we model the phonon action. We choose a model of $1 \mathrm{D}$ acoustic phonons linearly coupled to the electron density, assuming the phonon spectrum to be linear with a cutoff at the Debye frequency, $\omega_{\mathrm{D}}=c q_{\mathrm{D}}$. Then the phonon action (neglecting possible electron backscattering which is justified for $T \ll \omega_{D}$ ) has the following form:

$$
S_{\mathrm{el}-\mathrm{ph}}=\int \mathrm{i} \xi\left[-\frac{1}{2} \phi(\xi) \mathcal{D}_{0}^{-1} \phi(\xi)+g \phi(\xi) n(\xi)\right] .
$$

Here $\phi(\xi)$ is the phonon field, $g$ the el-ph coupling constant and $\mathcal{D}_{0}(\xi)$ the free phonon propagator with the Fourier transform given by

$$
\mathcal{D}_{0}(\omega, q)=\frac{\omega_{q}^{2}}{\omega^{2}-\omega_{q}^{2}+\mathrm{i} \delta}, \quad \omega_{q}=c q
$$

Finally, the tunnelling action for both geometries (assuming that the impurity or QD carrying the resonant level is inserted in the LL or side-attached to it at $x=0$ ) has the form:

$$
S_{\mathrm{T}}=\int \mathrm{i} t\left\{\mathrm{i} \bar{d}(t)\left(\partial_{t}+\varepsilon_{0}\right) d(t)+\sum_{\mu}\left[t_{0} \bar{d}(t) \psi_{\mu}(t)+\text { h.c. }\right]\right\},
$$

where $\psi_{\mu}(t) \equiv \psi_{\mu}(x=0, t), d(t)$ is the field corresponding to the electron localized at the resonant level with the energy $\varepsilon_{0}$ counted from the Fermi level. In the sideattached geometry (SAG) the index $\mu$ simply labels rightand left-movers while in the resonant-barriers geometry (RBG) it refers to the left and right electron subsystems separated by the barrier. In this case the electron can leave the left subsystem to the QD and enter it from the QD only as a right- and left-mover, respectively and conversely for the right subsystem so that the labels $\mu= \pm 1$ mean

$$
\bar{\psi}_{-}=\bar{\psi}_{\ell, L}, \psi_{-}=\psi_{\ell, R}, \bar{\psi}_{+}=\bar{\psi}_{r, R}, \psi_{+}=\psi_{r, L}
$$

where $L, R$ refer to the left- and right movers, as before, and $\ell, r$ to the left and right subsystems. We have assumed the tunnelling amplitude $t_{0}$ to be the same in all channels. This is always the case for the SAG, but not necessarily for the RBG if it is implemented as an asymmetric double-barrier. In the latter case the resonant properties could be different for $t_{1} \neq t_{2}$ but we leave it aside here.

The action defined by Eqs. (1), (3) and (4) is quadratic in fields $d$ and $\phi$ which can thus be integrated out. The integration over the phonon fields results only in substituting $V_{0}$ in the action (1) by the dynamical coupling,

$$
V(\xi)=V_{0}+g^{2} \mathcal{D}_{0}(\xi) .
$$

Performing the integration over the field $d(t)$ results in transforming the tunnelling term (4) in the action to the following one:

$$
\begin{gathered}
\tilde{S}_{\mathrm{T}}=-\sum_{\mu, \nu} \int \mathrm{i} t \mathrm{it}^{\prime} \bar{\psi}_{\mu}(t) \Sigma_{\mu, \nu}\left(t-t^{\prime}\right) \psi_{\nu}\left(t^{\prime}\right), \\
\hat{\Sigma}(\varepsilon)=\frac{v_{\mathrm{F}} \hat{\Gamma}_{0}}{\varepsilon-\varepsilon_{0}+\mathrm{i} \delta \operatorname{sgn} \varepsilon}, \quad \hat{\Gamma}_{0} \equiv \Gamma_{0}\left(\hat{1}+\hat{\sigma_{x}}\right),
\end{gathered}
$$

where $\Sigma\left(t-t^{\prime}\right)$ is the Fourier transform of $\Sigma(\varepsilon) . \hat{\Gamma}_{0}$ is the matrix with all elements equal to the tunnelling rate $\Gamma_{0} \equiv$ $\pi \nu_{0}\left|t_{0}\right|^{2}$, where $\nu_{0}=\left(\pi v_{\mathrm{F}}\right)^{-1}$ is the one-particle DoS of the conduction electrons in the absence of interactions.

The action given by Eqs. (1) (with $V_{0}=0$ ) and (7) describes the resonant transmission through the Fermi gas hybridized with the resonant level. The hybridization makes the electron Green function to acquire an off-diagonal part, $g_{\mu}\left(\xi-\xi^{\prime}\right) \rightarrow G_{\mu \nu}\left(\xi, \xi^{\prime}\right)$, describing 
the resonance-induced backscattering in the SAG or leftto-right connection in the RBG. In the mixed positionenergy representation it has the following matrix form

$$
\hat{G}\left(x, x^{\prime} ; \varepsilon\right)=\hat{g}\left(x-x^{\prime} ; \varepsilon\right)+\mathrm{i} v_{\mathrm{F}} \hat{g}(x ; \varepsilon) \hat{\mathrm{T}}(\varepsilon) \hat{g}\left(-x^{\prime} ; \varepsilon\right) .
$$

Here $\hat{g}$ is the matrix with diagonal elements given by eq. (2), and the $\hat{\mathrm{T}}$-matrix has the form

$$
\hat{\mathrm{T}}(\varepsilon)=\frac{-\mathrm{i} \hat{\Gamma}_{0}}{\varepsilon-\varepsilon_{0}+\frac{\mathrm{i}}{2} \hat{\Gamma}_{0} \operatorname{sgn} \varepsilon} .
$$

Although the Green function (8) is formally the same for both geometries considered, the transmission for the RBG is proportional to $G_{12} G_{21}$ while for the SAG it is proportional to $G_{11} G_{22}$. This gives the well-known Fermi-gas result with the resonant transmission for the RBG and the resonant reflection for the SAG (with $\left.\mathcal{R}_{0}=1-\mathcal{T}_{0}\right)$ :

$$
\mathcal{T}_{0}(\varepsilon)=\left\{\begin{array}{l}
\frac{\Gamma_{0}^{2}}{\left(\varepsilon-\varepsilon_{0}\right)^{2}+\Gamma_{0}^{2}}, \text { RBG } \\
\frac{\left(\varepsilon-\varepsilon_{0}\right)^{2}}{\left(\varepsilon-\varepsilon_{0}\right)^{2}+\Gamma_{0}^{2}}, \text { SAG } .
\end{array}\right.
$$

We will use the effective action represented by the sum of the terms given by eq. (1) with the substitution (6) and eq. (7) to show that the el-el and el-ph interactions results in $\Gamma_{0} \rightarrow \Gamma(\varepsilon)$ in the transmission probability (10), with the energy dependence of $\Gamma(\varepsilon)$ being qualitatively different from that found in the phononless case [9, 10].

\section{FUNCTIONAL BOSONIZATION}

The first step is the Hubbard-Stratonovich transformation which decouples the $n^{2}$ term in the action (1) with the substitution (6) and results in the mixed fermionicbosonic action in terms of the auxiliary bosonic field $\varphi$ minimally coupled to $\psi$ :

$$
S_{\text {eff }}=-\frac{1}{2} \int \mathrm{i} \xi \varphi V^{-1} \varphi+\mathrm{i} \sum_{\eta= \pm 1} \int \mathrm{i} \xi \bar{\psi}_{\eta}\left(\partial_{\eta}-\varphi\right) \psi_{\eta} .
$$

Here $\eta$ labels right- and left-moving electrons for both geometries under consideration. We stress that for the RBG there is no interaction between electrons in different halves of the system and in this case the action (11) describes electrons moving in one of the subsystems which are connected only via the resonant tunnelling. We will refer to electrons in different $(\ell$ and $r$ ) subsystems using indices $\mu, \nu= \pm 1$ which simultaneously label right- and left-movers as in eq. (5), keeping $\eta, \eta^{\prime}$ for referring only to the right- and left-movers in both geometries.

The introduction of the field $\varphi$ in eq. (11) does not constitute the functional bosonization: the latter is in getting rid of the coupling term by the following gauge transformation which introduces the new bosonic field $\theta(\xi) \equiv \theta(x, t)$ :

$$
\psi_{\eta}(\xi) \rightarrow \mathrm{e}^{\mathrm{i} \theta_{\eta}(\xi)} \psi_{\eta}(\xi), \quad \mathrm{i} \partial_{\eta} \theta_{\eta}(\xi)=\varphi(\xi) .
$$

The Jacobian of this transformation results [16] in substituting $V^{-1}+\Pi$ for $V^{-1}$ in eq. 11), where $\Pi=\Pi_{\mathrm{R}}+\Pi_{\mathrm{L}}$ is the one-loop electronic polarization operator (exact for the LL), with $\Pi_{\eta}(\xi)=\mathrm{i} g_{\eta}(\xi) g_{\eta}(-\xi)$ and $g_{\eta}(\xi)$ given by eq. (2). Note that one could have started with the unscreened Coulomb interaction, as this transformation provides the screening which is, naturally, identical to one originally calculated diagrammatically [17].

The conduction electron Green function is not invariant under the gauge transform 12 and so it is dressed by $\theta(\xi)$. In the absence of the resonance part of the action, eq. (7), the dressed Green function for right- or left-moving electrons is given by

$$
G_{\eta}\left(\xi-\xi^{\prime}\right)=g_{\eta}\left(\xi, \xi^{\prime}\right) \mathrm{e}^{\mathrm{i} U_{\eta \eta}\left(\xi, \xi^{\prime}\right)},
$$

where $\mathrm{i} U_{\eta \eta^{\prime}} \equiv\left\langle\theta_{\eta} \theta_{\eta^{\prime}}\right\rangle$ is the correlation function of the fields $\theta$. To calculate it we notice that eq. 12 for the gauge field $\theta(\xi)$ is resolved with the help of the bosonic Green function $g_{\eta}$ which coincides with the free electron Green function (2):

$$
\theta_{\eta}(\xi)=\int \mathrm{i} \xi^{\prime} g_{\eta}\left(\xi-\xi^{\prime}\right) \varphi\left(\xi^{\prime}\right)
$$

Thus we obtain the Fourier transform of $U_{\eta \eta^{\prime}}$ as follows:

$$
U_{\eta \eta^{\prime}}(\omega, q)=\frac{\omega_{+}+\eta^{\prime} v_{\mathrm{F}} q}{\omega_{+}-\eta v_{\mathrm{F}} q} \frac{V_{0}\left(\omega_{+}^{2}-\omega_{q}^{2}\right)+g^{2} \omega_{q}^{2}}{\left(\omega_{+}^{2}-v_{+}^{2} q^{2}\right)\left(\omega_{+}^{2}-v_{-}^{2} q^{2}\right)},
$$

where $\omega_{+} \equiv \omega+\mathrm{i} \delta \operatorname{sgn} \omega$ and $v_{ \pm}$are velocities of the composite bosonic modes (polarons) given by

$$
v_{ \pm}^{2}=\frac{1}{2}\left[v^{2}+c^{2} \pm \sqrt{\left(v^{2}-c^{2}\right)^{2}+4 \alpha_{\mathrm{ph}} v_{\mathrm{F}}^{2} c^{2}}\right] .
$$

Here we introduced the dimensional el-ph coupling constant $\alpha_{\mathrm{ph}} \equiv \nu_{0} g^{2}$ while $v$ is the speed of plasmonic excitations in the phononless LL,

$$
v=v_{\mathrm{F}}\left(1+\nu_{0} V_{0}\right)^{1 / 2} \equiv v_{\mathrm{F}} K^{-1},
$$

where $K$ is the standard Luttinger parameter. We assume that in the absence of phonons the el-el interaction is always repulsive $\left(V_{0}>0\right)$ so that $K<1$. Note that in the limit corresponding to the absence of phonons $(c=0)$, one has $v_{-}=0$ and $v_{+}=v$, so that in this case $U_{\mu \nu}$ reduces to the usual LL plasmonic propagator. This also happens in the absence of the el-ph coupling $\left(\alpha_{\mathrm{ph}}=0\right)$ when $v_{-}=\min (c, v)$ and $v_{+}=\max (c, v)$ and these two branches are totally decoupled. We take the same limit for $U$ when $\omega>\omega_{\mathrm{D}}$ so that $c$ should be put to 0 . 
Equations (13) and 14 describe well known twobranch polaronic excitations [12] in the LL in the presence of the el-ph coupling. The slow and fast branches have the velocity $v_{\mp}$ obeying the inequalities $v_{-}<c, v<$ $v_{+}$. Here we have restricted our considerations to the stability region defined by

$$
\alpha \equiv \alpha_{\mathrm{ph}} K^{2}<1,
$$

where $v_{-}^{2}>0$, i.e. we leave out of considerations the Wentzel-Bardeen instability [18].

The existence of the two polaron branches may be interpreted as splitting the LL in the presence of the el-ph coupling into the two-component liquid, with the effective Luttinger parameters $K_{\text {fast }}=v_{\mathrm{F}} / v_{+}<K<$ $1, \quad K_{\text {slow }}=v_{\mathrm{F}} / v_{-}>1$, corresponding to el-el repulsion (which becomes stronger with the el-ph coupling) and the phonon-mediated attraction. The two-mode nature of the el-ph LL drastically changes a character of the resonant (or antiresonant) transmission.

The self-energy part in resonant-level term (7) is dressed as a result of the gauge transform (12) by the local field $\theta(\varepsilon) \equiv \theta(x=0, t)$ :

$$
\Sigma\left(t-t^{\prime}\right) \rightarrow \Sigma_{\mu \nu}\left(t-t^{\prime}\right)=\mathrm{e}^{-\mathrm{i} \theta_{\mu}(t)} \Sigma\left(t-t^{\prime}\right) \mathrm{e}^{\mathrm{i} \theta_{\nu}\left(t^{\prime}\right)} .
$$

It is this dressing which fully governs the resonance-width renormalization, $\Gamma_{0} \rightarrow \Gamma(\varepsilon)$, in the presence of the el-el and el-ph interactions.

\section{THE SELF-ENERGY RENORMALIZATION}

The polaron fields $\theta_{\mu}(t)$ entering the self-energy 16 are defined at the origin where the QD (or barriers) carrying the resonant level is placed. Integrating out all the fields at $x \neq 0$ results in the zero-dimensional action which governs the renormalization of the self-energy in Eqs. (7) and (16), and thus the renormalization of the resonance width in Eqs. 101. In the phononless case this action is fully equivalent to that used for describing the resonant transmission (reflection) through the LL [9, 10, . The only difference due to the el-ph coupling is that the correlation function of the local fields $\theta(t)$ is governed by the two-branch polaron modes, eq. 13 . It follows from this equation that

$$
\left\langle\theta_{\mu}(-\omega) \theta_{\nu}(\omega)\right\rangle=\mathrm{i} \int \frac{\mathrm{i} q}{2 \pi} U_{\mu \nu}(\omega ; q) \equiv \frac{\pi \gamma_{\mu \nu}}{|\omega|},
$$

where the dimensionless correlation matrix $\gamma_{\mu \nu}$ found from the above integration can be parameterized as

$$
\gamma_{\mu \nu}= \begin{cases}\delta_{\mu \nu} \gamma_{+}, & \text {RBG } \\ \frac{1}{2}\left(\gamma_{+}-\gamma_{-}\right)+\gamma_{-} \delta_{\mu \nu}, & \text { SAG. }\end{cases}
$$

Here $\gamma_{ \pm}$can be represented as

$$
\gamma_{+}=\frac{\kappa_{+}}{K}-1, \quad \gamma_{-}=\kappa_{-} K-1,
$$

where one would have $\kappa_{ \pm}=1$ without coupling to phonons in which case $\gamma_{+} \rightarrow \tilde{\gamma}_{+} \equiv 1 / K-1$, the exponent describing renormalization of the conductance by a weak scatterer, and $\gamma_{-} \rightarrow \tilde{\gamma}_{-} \equiv K-1$, the exponent describing renormalization by a weak link. The el-ph coupling modifies these exponents by the factors $\kappa_{ \pm}(K)$ given by

$$
\begin{aligned}
& \kappa_{+}=\left[1+\frac{\alpha}{(\beta+\sqrt{1-\alpha})^{2}}\right]^{-1 / 2}, \quad \beta \equiv \frac{v}{c}, \\
& \kappa_{-}=\left\{(1-\alpha)\left[1+\frac{\alpha}{\left(\beta^{-1}+\sqrt{1-\alpha}\right)^{2}}\right]\right\}^{-1 / 2} .
\end{aligned}
$$

It is easy to verify that $\kappa_{+}(K) \leq 1$ while $\kappa_{-}(K) \geq$ 1 , so that $\gamma_{+}<\tilde{\gamma}_{+}$and $\gamma_{-}>\tilde{\gamma}_{-}$. Therefore, whereas the phononless exponents are sign-definite, $\tilde{\gamma}_{+}>0$ and $\tilde{\gamma}_{-}<0$, this is not necessarily true for the exponents $\gamma_{+}$ and $\gamma_{-}$. We have previously shown [15] that electronic transport through the LL with a weak scatter or with a weak link, described by the correlation functions with the exponents $\gamma_{ \pm}$respectively, is strongly influenced by the el-ph coupling due to these exponents changing sign at different values of parameters $\alpha$ and $\beta$. Here we will show that this also strongly affects the resonant transmission (reflection) in both geometries where the exponents $\gamma_{ \pm}$ are changed by the el-ph interaction enter via eq. (18).

As in the case of the resonance transport through the phononless LL 9, 10, we shall write a renormalization group (RG) equation for the tunnelling amplitude $t_{0}$. In our formalism we should start with renormalizing the self-energy part $\Sigma$ in the tunnelling action (7) with the gauge substitution (16).

Having integrated out the fields with $x \neq 0$, all the interaction effects enter via the correlation functions of the bosonic field $\theta$, defined by Eqs. (17) - 20). Therefore, the RG equation for $\Sigma$ is obtained by a usual integration over fast components of this field. We do not integrate over fast components of the fermionic field $\psi(t)$ and do not rescale the time variable since these two procedures exactly cancel each other which follows from the absence of the renormalization of the self-energy in the noninteracting case. We assume that the fast Fourier components of the field $\theta_{\mu}(t)$ have frequencies $E \leq|\omega| \leq E^{\prime}$, with $E$ being the running cutoff and $E^{\prime} / E-1 \ll 1$. Integrating them out leads to the following increment for the selfenergy:

$$
\begin{aligned}
\delta \Sigma_{\mu \nu}(\varepsilon)=-\int_{E \leq|\omega| \leq E^{\prime}} \frac{\mathrm{d} \omega}{2|\omega|}[ & \frac{\gamma_{\mu \mu}+\gamma_{\nu \nu}}{2} \Sigma_{\mu \nu}(\varepsilon) \\
& \left.-\gamma_{\mu \nu} \Sigma_{\mu \nu}(\varepsilon+\omega)\right] .
\end{aligned}
$$

In such an RG scheme [9] the self-energy $\Sigma_{\mu \nu}$ acquires a dependence on the running cutoff $E$ on top of the dependence on $\varepsilon$. The initial condition for the RG equations 
is that at the ultraviolet cutoff, $E=E_{0},\left(E_{0} \sim \varepsilon_{\mathrm{F}}\right.$ is the bandwidth), $\Sigma_{\mu \nu}$ is independent of $E$ and has all matrix elements equal to $\Sigma(\varepsilon)$ given by eq. (7). Then as long as $E \gg\left|\varepsilon-\varepsilon_{0}\right|$ one may discard the second term in eq. (21), thus arriving at the following RG equation:

$$
\frac{\mathrm{d} \Sigma_{\mu \nu}(\varepsilon ; E)}{\mathrm{d} l}=-\gamma_{\mathrm{d}} \Sigma_{\mu \nu}(\varepsilon ; E),
$$

where $l \equiv \ln E_{0} / E$ and $\gamma_{\mathrm{d}}$ are equal diagonal components of the matrix $\gamma_{\mu \nu}$, eq. 18, given by

$$
\gamma_{\mathrm{d}}=\left\{\begin{array}{cc}
\gamma_{+}, & \text {RBG } \\
\gamma_{0} \equiv \frac{1}{2}\left(\gamma_{+}+\gamma_{-}\right), & \text {SAG }
\end{array}\right.
$$

Note that $\gamma_{+}$and $\gamma_{0}$ happen to be the edge and bulk DoS exponents respectively, equal to $1 / K-1$ and $(1-K)^{2} / 2 K$ in the phononless case [5] and given by eq. (18) and (20) in the presence of the el-ph interaction.

Equation $(22)$ is solved by substituting $\Sigma_{\mu \nu}(\varepsilon ; E)$ in form (7), i.e. with all matrix elements equal, but with $\Gamma_{0}$ replaced by $\Gamma(E)$. This leads to the RG equation for $\Gamma$ :

$$
\frac{\mathrm{d} \Gamma(E)}{\mathrm{d} l}=-\gamma_{\mathrm{d}} \Gamma(E), \quad E \gg\left|\varepsilon-\varepsilon_{0}\right| .
$$

With lowering the running cutoff one eventually reaches the region $E \ll\left|\varepsilon-\varepsilon_{0}\right|$ where the second term must be taken into account. The self-energy still has the form of eq. (7) but with the substitution

$$
\hat{\Gamma}_{0} \rightarrow \hat{\Gamma}(E)=\Gamma_{\text {diag }}(E) \hat{1}+\Gamma_{\text {off }}(E) \hat{\sigma}_{x} .
$$

For $E \ll\left|\varepsilon-\varepsilon_{0}\right|$ the second term in eq. (21) cancels the first one for $\mu=\nu$ so that $\Gamma_{\text {diag }}$ saturates at $\Gamma\left(\left|\varepsilon-\varepsilon_{0}\right|\right)$ obtained by solving eq. 24, while $\Gamma_{\text {off }}$ continues to be renormalized according to the following RG equation:

$$
\frac{\mathrm{d} \Gamma_{\text {off }}(E)}{\mathrm{d} l}=-\gamma_{ \pm} \Gamma_{\text {off }}(E), \quad E \ll\left|\varepsilon-\min \left\{\Gamma_{0}, \varepsilon_{0}\right\}\right|,
$$

with $\gamma_{+}$for the RBG, as in eq. 24, and $\gamma_{-}$for the SAG. Behavior of renormalized $\Gamma(\varepsilon)$ is shown in fig. 2 . Let us stress that the condition of applicability written above does not follow directly from eq. 21) which is perturbative in $\Gamma_{0}$. However, it follows from considerations non-perturbative in tunnelling (but perturbative in the interaction strength) [6, 10, that the inequality (26) actually means the off-resonance condition. The impurity remains off-resonant if the level width renormalized according to eq. 24) remains narrow, i.e. $\Gamma\left(\varepsilon_{0}\right) \ll \varepsilon_{0}$. Only in this case $\Gamma_{\text {off }}$ renormalizes as in eq. (26). Otherwise, we should put $\varepsilon_{0}=0$ and describe the resonant situation entirely in the frame of eq. (24).

\section{TRANSMISSION COEFFICIENT}

The transmission coefficient, $\mathcal{T}(\varepsilon)$, is obtained by replacing in the $\hat{\mathrm{T}}$-matrix $(9)$ the bare tunneling rates, $\hat{\Gamma}_{0}$,

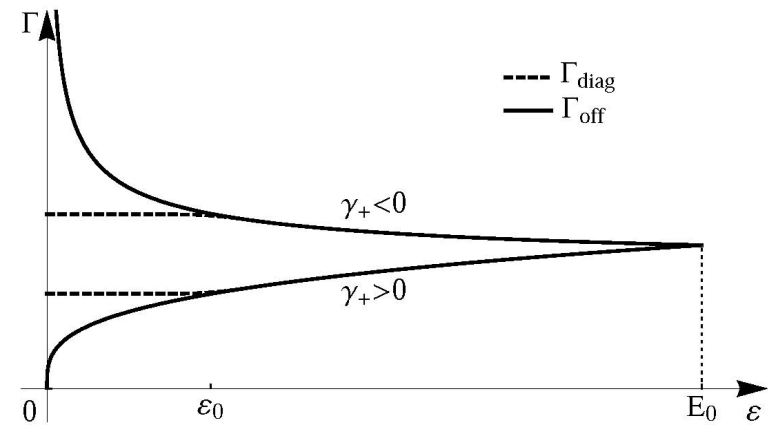

(a)Resonant-barrier geometry: $\Gamma_{\text {off }} \propto\left|\varepsilon / E_{0}\right|^{\gamma+}$ with the sign of $\gamma_{+}$depending on the el-ph coupling strength.

$\Gamma_{\text {diag }}=\Gamma_{\text {off }}$ at $\varepsilon \gtrsim \varepsilon_{0}$ and saturates at $\varepsilon_{0}$ (dashed line) for $\varepsilon \lesssim \varepsilon_{0}$

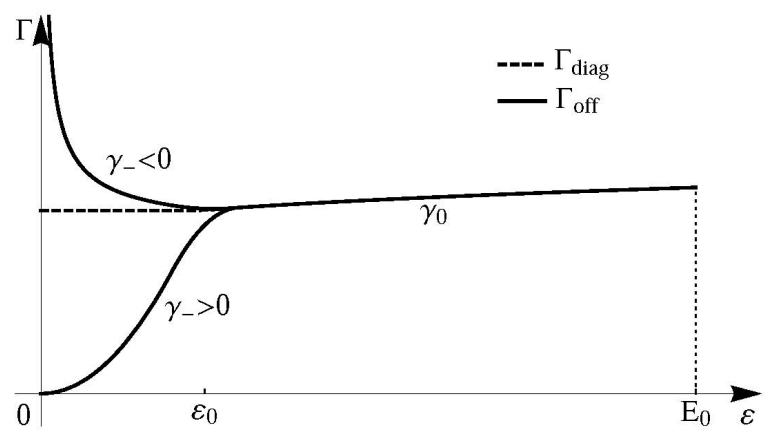

(b)Side-attached geometry: $\Gamma_{\text {diag }}=\Gamma_{\text {off }} \propto\left|\varepsilon / E_{0}\right|^{\gamma_{0}}$ for $\varepsilon \gtrsim \varepsilon_{0} ; \Gamma_{\text {diag }}$ saturates and $\Gamma_{\text {off }} \propto\left|\varepsilon / \varepsilon_{0}\right|^{\gamma-}$ for $\varepsilon \lesssim \varepsilon_{0}$. The sign of $\gamma_{-}$depends on the el-ph coupling strength, while $\gamma_{0}>0$.

FIG. 2. $\Gamma_{\text {diag }}(\varepsilon)$ and $\Gamma_{\text {off }}(\varepsilon)$ for RBG and SAG (not to scale).

by the renormalized ones, $\hat{\Gamma}(E=\varepsilon)$, found from Eqs. 24 and (26). The off-diagonal element of the transmission matrix, $\mathrm{T}_{\text {off }}$, is equal to the transmission or reflection amplitude for, respectively, the RBG or SAG so that

$$
\mathcal{T}(\varepsilon)=\left\{\begin{array}{cl}
\left|\mathrm{T}_{\text {off }}(\varepsilon)\right|^{2}, & \text { RBG } \\
1-\left|\mathrm{T}_{\text {off }}(\varepsilon)\right|^{2}, & \text { SAG }
\end{array} .\right.
$$

Here $\left|\mathrm{T}_{\text {off }}(\varepsilon)\right|^{2}$ has the following form found from eq. (9) with the substitution 25) at $E=\varepsilon$ :

$$
\left|\mathrm{T}_{\mathrm{off}}\right|^{2}=\frac{\Gamma_{\mathrm{off}}^{2}}{\left(\varepsilon_{0}-\varepsilon+\Lambda\right)^{2}+\Gamma_{\mathrm{diag}}^{2}}, \quad \Lambda \equiv \frac{\Gamma_{\mathrm{off}}^{2}-\Gamma_{\mathrm{diag}}^{2}}{4 \varepsilon_{0}} .
$$

For $\varepsilon \gtrsim\left|\varepsilon_{0}\right|$, the diagonal and off-diagonal elements of $\hat{\Gamma}$ are equal and renormalize in the same way, eq. 24. This leads to the transmission coefficients of the Fermigas form 10 but with the renormalized tunneling rate,

$$
\Gamma(\varepsilon)=\Gamma_{0}\left(\frac{\varepsilon}{E_{0}}\right)^{\gamma_{\mathrm{d}}}
$$

which fully describes the case of resonance. When the impurity level is off-resonance, we substitute $\varepsilon_{0}$ for $\varepsilon_{0}-\varepsilon$ 


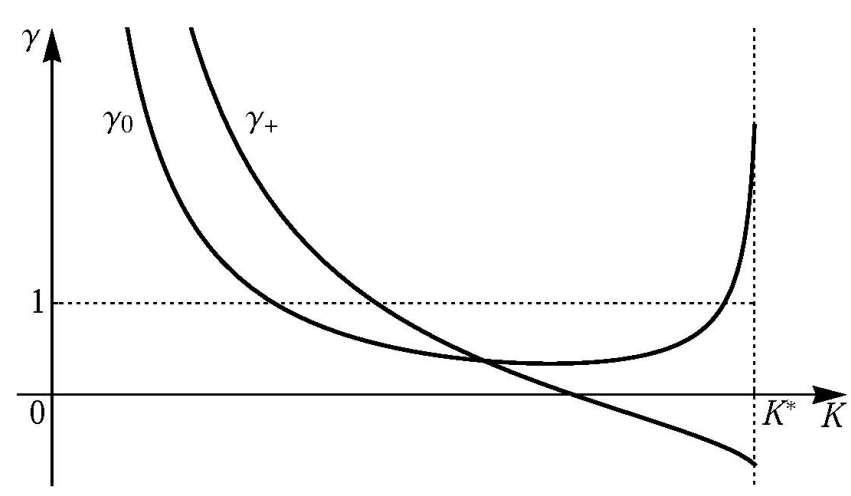

FIG. 3. The RG exponents of the effective resonance width for the RBG, $\gamma_{+}$, and the SAG, $\gamma_{0}$. Here $K^{*} \equiv \min \left\{1, \alpha_{\mathrm{ph}}{ }^{-\frac{1}{2}}\right\}$ is the boundary of the applicability region: for $\alpha_{\mathrm{ph}}>1$ we stay away from the Wentzel-Bardeen instability [18].

into eq. (28) and take into account that $\Gamma_{\text {off }}$ continues to renormalize, eq. 26 , while $\Gamma_{\text {diag }}$ saturates:

$$
\Gamma_{\text {off }}(\varepsilon)=\Gamma\left(\varepsilon_{0}\right)\left(\frac{\varepsilon}{\varepsilon_{0}}\right)^{\gamma_{ \pm}}, \quad \Gamma_{\text {diag }}=\Gamma\left(\varepsilon_{0}\right) .
$$

\section{RESONANT CONDUCTANCE}

At nonzero but low temperatures $T$, the two-terminal conductance $g(T)$ is proportional to $\mathcal{T}(\varepsilon)$ with the lowenergy cutoff at $\varepsilon \sim T$ (the Fermi energy corresponds to $\varepsilon=0)$. In the off-resonance situation, $\left|\mathrm{T}_{\text {off }}(\varepsilon)\right|$ in eq. 28) vanishes with $\varepsilon \rightarrow 0$ : the resonant level remains decoupled from conduction electrons even when $\Gamma_{\text {off }}(\varepsilon)$ diverges when $\varepsilon \rightarrow 0$.

The resonance, $\varepsilon_{0} \lesssim \Gamma\left(\varepsilon_{0}\right)$, is described by eq. (28) with $\Lambda=0$. This corresponds to the Fermi-gas expression, eq. 110, with $\varepsilon_{0}=0$ and $\Gamma_{0}$ substituted by its renormalized value, $\Gamma(\varepsilon)$, eq. $(29)$. The critical exponent $\gamma_{\mathrm{d}}$, eq. (23), is strongly affected by the el-ph coupling, as illustrated in fig. 3; without phonons $\gamma_{\mathrm{d}}$ (i.e. $\gamma_{+}$or $\gamma_{0}$ ) are monotonically decreasing functions reaching 0 at $K=1$. Crucially, conductance $g$ is either ideal or vanishing at $T=0$, depending on whether $\gamma_{\mathrm{d}}>1$ or $\gamma_{\mathrm{d}}<1$.

For $\gamma_{\mathrm{d}}>1$, $\left|\mathrm{T}_{\text {off }}(\varepsilon)\right|$ in eq. 28) vanishes with $\varepsilon \rightarrow 0$, as in the case of a strong el-el coupling in the phononless LL $(K<1 / 2$ without pair tunnelling [5]). This happens because $\Gamma(\varepsilon) \rightarrow 0$ faster than $\varepsilon \rightarrow 0$, i.e. the resonant level remains effectively decoupled from conduction electrons.

On the contrary, for $\gamma_{\mathrm{d}}<1$ we have $\left|\mathrm{T}_{\text {off }}(\varepsilon)\right| \rightarrow 1$ for $\varepsilon \rightarrow 0$ which leads to an ideal resonance for the RBG and antiresonance for the SAG, eq. (27).

Thus the effective decoupling of the resonant electron level from conduction electrons at $\gamma_{\mathrm{d}}=1$ leads to a metal-insulator transition. Figure 3 shows that for the RBG, where $\gamma_{\mathrm{d}} \equiv \gamma_{+}$, the el-ph coupling shifts the transition towards stronger el-el coupling. Note also that when $\gamma_{+}<0$, the effective resonance width diverges with $\varepsilon \rightarrow 0$ rather than vanishes as in the phononless LL. For the SAG, where $\gamma_{\mathrm{d}} \equiv \gamma_{0}$, there could be two phase transitions: a sufficiently strong el-ph interaction can decouple the resonant level from conduction electrons, leading to the metallic phase, also for a weak el-el coupling when $K$ is close to 1.

This work was supported by the EPSRC Grant $\mathrm{T} 23725 / 01$.

[1] Tomonaga S., Prog. Theor. Phys., 5 (1950) 544; LutTinger J. M., J. Math. Phys., 4 (1963) 1154; Haldane F. D. M., J. Phys. C, 14 (1981) 2585; von Delft J. and Schoeller H., Ann. Phys., 7 (1998) 225.

[2] Bockrath M. et al., Nature, 397 (1999) 598; YAO Z. et al., ibid, 402 (1999) 273; IsHII H. et al., ibid, 426 (2003) 540; LeE J. et al., Phys. Rev. Lett., 93 (2004) 166403.

[3] Auslaender O. et al., Science, 295 (2002) 825; Slot E. et al., Phys. Rev. Lett., 93 (2004) 176602; Levy E. et al., ibid, 97 (2006) 196802; Venkataraman L., Hong Y. S. and Kim P., ibid, 96 (2006) 076601.

[4] Deshpande V. V., Bockrath M., Glazman L. I. and YACOBY A., Nature, 464 (2010) 209.

[5] Kane C. L. and Fisher M. P. A., Phys. Rev. Lett., 68 (1992) 1220; Phys. Rev. B, 46 (1992) 15233.

[6] Matveev K. A., Yue D. and Glazman L. I., Phys. Rev. Lett., 71 (1993) 3351.

[7] Furusaki A., Phys. Rev. B, 56 (1997) 9352.

[8] Egger R. and Grabert H., Phys. Rev. Lett., 75 (1995) 3505; White S. R., Affleck I. and Scalapino D. J., Phys. Rev. B, 65 (2002) 165122.

[9] Kane C. L. and Fisher M. P. A., Phys. Rev. B, 46 (1992) 7268(R); Polyakov D. G. and Gornyi I. V., ibid, 68 (2003) 035421; Furusaki A. and Matveev K. A., Phys. Rev. Lett. , 88 (2002) 226404; NAzArov Y. V. and Glazman L. I., ibid, 91 (2003) 126804.

[10] Lerner I. V., Yudson V. I. and Yurkevich I. V., Phys. Rev. Lett., 100 (2008) 256805; Goldstein M. and Berkovits R., ibid, 104 (2010) 106403.

[11] Bockrath M. et al., Science, 291 (2001) 283; MASon N., Biercuk M. J. and Marcus C. M., ibid, 303 (2004) 655; Terrones M. et al., Phys. Rev. Lett., 89 (2002) 075505.

[12] Loss D. and Martin T., Phys. Rev. B, 50 (1994) 12160.

[13] Cazalilla M. A. and Ho A. F., Phys. Rev. Lett., 91 (2003) 150403; Mathey L., Wang D.-W., HofstetTER W., Lukin M. D. and Demler E., ibid, 93 (2004) 120404.

[14] San-Jose P., Guinea F. and Martin T., Phys. Rev. $B, 72$ (2005) 165427.

[15] Galda A., Yurkevich I. V. and Lerner I. V., Impurity scattering in Luttinger liquid with electron-phonon coupling, arXiv:1008.3270 (2010).

[16] Grishin A., Yurkevich I. V. and Lerner I. V., Phys. Rev. B, 69 (2004) 165108.

[17] Dzyaloshinskit I. E. and Larkin A. I., Zh. Eksp. Teor. Phys., 65 (1973) 411.

[18] Wentzel G., Phys. Rev., 83 (1951) 168; Bardeen J., Rev. Mod. Phys., 23 (1951) 261. 